\title{
Probability distribution of orbital crossing times in a protoplanetary system
}

\author{
Hiroyuki Emori ${ }^{1}$, Kiyoshi Nakazawa ${ }^{2}$, and Kazunori Iwasaki ${ }^{3}$ \\ ${ }^{1}$ Shumei University, Daigaku-Cho, Yachiyo-city, Chiba Prefecture, Japan \\ ${ }^{2}$ Tokyo Institute of Technology, 1-12-1 Oookayama, Meguro-ku, Tokyo, Japan \\ ${ }^{3}$ Laboratory for Atomospheric and Space Physics, Univ. Colorado, 392, UCB Boulder, CO 80309-0392, USA
}

(Received June 4, 2007; Revised November 8, 2007; Accepted January 10, 2008; Online published July 4, 2008)

\begin{abstract}
Long term behavior of five protoplanets was studied under the same conditions as those used in Chambers et al. (1996). One major difference was the number of calculations carried out for one parameter set of initial orbital distance. We reconfirmed their result for the orbital crossing times among five protoplanets starting from circular and co-planar orbits with an equal distance of their semi major axes. For each distance, the distribution of orbital crossing times was calculated from 500 sets of azimuthal positions of protoplanets randomly chosen. The distribution of the times around the average value resembles each other for almost all orbital distance cases. Based on a statistical certification we conclude that the fluctuations in orbital crossing times take "the log normal function". The dispersion of the log normal distribution function is equal to 0.2 . This means that $70 \%$ of the events of orbital crossing occurs in the range between $10^{-0.2}$ times earlier and $10^{0.2}$ times later than the average orbital crossing time.
\end{abstract}

Key words: Protoplanet, orbital crossing, planetary formation.

\section{Introduction}

In the last decade we have made major advances in studies of planetary formation processes. This is especially true in terms of the accumulation of small planetesimals; we now have the key to shorten time to build a protoplanet. This is "Run away growth" originally proposed by Wetherill and Stewart (1989). In the swarm of planetesimals, energy equipartition between bodies are established. This statistical mechanism determines the average random velocities as a decreasing function of mass of planetesimals. This is well known as "dynamical friction" in the swarm (Stewart and Wetherill, 1988). Under the effect of the dynamical friction, the random velocity of protoplanets is suppressed to be much smaller than that of field planetesimals (Stewart and Wetherill, 1988). In contrast, the random velocity of planetesimal is almost equal to the escape velocity from one planetesimal. As a result, the relative velocity between a large protoplanet and a small-field planetesimal is much smaller than the escape velocity from the protoplanet and the ratio between these two velocities, which is called the "Safronov parameter" (Safronov, 1969), becomes larger as the protoplanet grows. This increase in Savronov parameter enhances the collisional cross section of protoplanets to planetesimals greatly, which is the run-away growth stage (Wetherill and Stewart, 1989).

Wetherill and Stewart (1989) were the first to propose the runaway growth, though they treated the swarm of planetesimal as "particles in a box", neglecting the effect of strong external solar gravity. Ida (1990) employed a sophisticated

Copyright (c) The Society of Geomagnetism and Earth, Planetary and Space Sciences (SGEPSS); The Seismological Society of Japan; The Volcanological Society of Japan; The Geodetic Society of Japan; The Japanese Society for Planetary Sciences; TERRAPUB theoretical formulation to treat gravitational scattering in the solar gravitational field which he called "Hill approximation" (Petit and Henon, 1987; Nakazawa and Ida, 1988), and confirmed that dynamical friction actually works, and the runaway growth as well, in a swarm of planetesimal revolving around the Sun. Ida estimates the accumulation time of moon sized protoplanets in the earth region at less than one million years, though some questions remain, such as "When does the runaway growth finish?", "How are the protoplanets distributed around the Sun after the process ceased?".

Kokubo and Ida (1998) were the first to study on the spacial distribution among planetesimal and protoplanets during the accumulation process. They carried out direct orbital calculations, which is called "N-body simulations", on the swarm of planetesimals. From their results, about ten protoplanets with about Martian mass were produced in the region of present-day Venus, Earth, and Mars orbits. In addition, the orbital distance between each protoplanet is almost equal. This distance is about 10 mutual hill radius. Protoplanets are arranged to revolve in circular orbits at an equal distance in semi major axes. This is a condition of protoplanets just after the runaway growth stage.

Chambers et al. (1996) studied the behavior of protoplanets starting from the initial condition of protoplanets. They put five or more Martian-sized protoplanets in the presentday Venus, Earth, and Mars region. Initially the radial distance between each orbit was set to be equal. The distance was an important parameter in their study, as it is in our study. They then started orbital calculations. During many orbital revolutions, protoplanets interact gravitationally with each other and increase their eccentricities. When a protoplanet reches an orbital eccentricity that is large enough to approach an adjoining protoplanet, the pair 
of protoplanets can encounter each other in their mutual Hill sphere (Petit and Henon, 1987; Nakazawa and Ida, 1988). Chambers et al. called this event "orbital crossing". Their aim was to determine time needed for this orbital crossing event as a function of orbital distance.

They put a parameter $\Delta$ to represent the initial orbital distance between each planet. They put five planets in five circular orbits around the sun. The difference in the semimajor axis between adjoining orbits was set to $\Delta$ times Hill radius, which is the radius of the mutual Hill sphere.

They pursued numerically the orbital evolution of planets with changing $\Delta$ from 3.6 to 8.0 , for every 0.2 . For each value of $\Delta$, five cases of initial azimuthal conditions among five planets were examined. The most important results of the Chambers et al. (1996) study are that the orbital crossing time depends on the orbital distance $\Delta$ exponentially. Actually, from figure 1 in their work or Fig. 2(c) in this paper, though a fitting line show some considerable discrepancies from data points, we can recognize that the orbital crossing times plotted logarithmically shows good linear correlation with $\Delta$.

Even if the initial orbital distance $\Delta$ is equal, the orbital crossing time becomes different owing to the difference in the initial azimuthal positions. Chambers et al. (1996) reported that we have about one order of magnitude difference between maximum and minimum orbital crossing time for identical values of $\Delta$, in some cases.

The orbital crossing time depends strongly on the initial orbital distance. Namely, unity increment in $\Delta$ results in about one order of magnitude elongation of the orbital crossing time. This result has a great importance on the planetary formation process. Because protoplanets were formed with distances about 10 Hill radius (Kokubo and Ida, 1998), adopting their results to this case, the orbital crossing time was determined to be over one billion years. This long interval necessary for orbital crossing among protoplanets could make the time for planetary formation longer than the life time of protoplanetary nebula gas which is about several million years (Strom et al., 1993; Strom, 1995). This raises some serious difficulties with the theory of Jovian planets formation (e.g. Mizuno et al., 1978; Ikoma et al., 2000). Owing to its importance to the study of planetary formation theories, the relation of Chambers et $a l$. has been studied by many authors after it was proposed.

One extension of Chambers law with breaking the initial condition that all protoplanets have zero eccentricity was attempted by Yoshinaga et al. (1999). They initially pursued orbital evolution of protoplanets which revolve around the sun along eccentric orbits and suggested that initial eccentricities make the orbital crossing time shorter than those given by the Chambers law.

A strong perturbing source, namely Jupiter could have a large effect on the evolution of protoplanets around the present orbit of the Earth. Therefore if the Jovian planet is accumulated earlier than terrestrial planets, we have to develop some new expressions on orbital crossing times. Because Chambers et al. did not take Jupiter into account, Ito and Tanikawa (1999) considered a new relation between initial orbital distance and orbital crossing time under the condition that there are already giant planets, namely Jupiter and Saturn. They presented two important results: one is that strong gravitational perturbation from the giant planets effected a secular evolution on the eccentricities of protoplanets and made the orbital crossing time shorter than those in the case of no giant planets, the second is that a protoplanet, especially the protoplanet revolving on the most outer orbit, could make an orbital crossing not only with other protoplanets but also with Jupiter. So the relation between orbital distance and the orbital crossing time should be changed in the specific case that the orbital distance is large enough to make the most outer protoplanet approach the giant planet easily.

As mentioned before planetary accretion processes have a strong relation to the random velocity of protoplanets and planetesimals. The random velocity of planetesimals and protoplanets are determined by the gravitational scattering between them and some kinds of dissipative forces. The gas drag force from protoplanetary nebula is the first candidate for the dissipative force on protoplanets (Adachi et al., 1978). Although Chambers et al. supposed the protoplanetary system in vacuum, it is a straightforward extension of Chambers et al. to consider the protoplanetary system in the gas nebula.

Iwasaki et al. (2001) studied the orbital crossing time for the protoplanetary systems using the same approach as Chambers et al. except that the former consider protoplanets revolving around the sun under the gas drag effects from protoplanetary nebula (e.g. Adachi et al., 1976). They studied the behavior of protoplanets under a type of gas drag force that is proportional to square of the random velocity, namely the Stokes-type gas drag. Gas drag force suppresses the eccentricities of protoplanets, as such, protoplanets can hardly approach another one revolving on the adjoining orbit. Iwasaki et al. (2001) presented a critical orbital distance $\Delta_{c}$ as a function of nebular gas densities that determine the strength of the gas drag force. In the case that the orbital distance $\Delta$ is smaller than this critical value $\Delta_{c}$, the protoplanet system has the orbital crossing event during a length of time which is almost equal to the orbital crossing time given in Chambers et al. (1996). On the other hand, if the orbital distance exceeds the critical distance, the protoplanet system does not have any orbital crossing event, at least during a period which is one hundred times longer than the orbital crossing time for the gas-free case.

In the same way, Iwasaki et al. (2002) studied the evolution of protoplanets under the effect of another type of gas drag force that is expressed to be proportional linearly to the random velocity. They also presented the critical orbital distance $\Delta_{c}$ in this case. For both cases of gas drag force the critical orbital distance $\Delta_{\mathrm{c}}$ is determined from three pieces of information: the first is the intensity of the drag force, the second is orbital crossing time in the gas-free case, the third is stirring rate of random velocity given from three bodies using the distant encounter formulation by Hasegawa and Nakazawa (1990), which is also formulated for the gas-free case. This formula is very useful to study the orbital instabilities among protoplanets in nebula gas.

These studies focused on modifying of the Chambers et al. (1996) relation taking into account some additional factors which should be considered in actual planetary for- 
mation processes. However, the most important question, 'Why does the Chambers relation exist?' has never been answered. The relation is a purely empirical law obtained from numerical experiments under some restricted initial conditions, namely zero eccentricities and equal orbital distance. So, every time we want to break the restriction on the initial conditions we have to again perform numerous orbital calculations on the protoplanetary system, as did Chambers et al.

In other words, we need a theoretical model on the orbital crossing time which can readily explain the Chambers relation under the condition that Chambers put in their work. This model would also allow us to estimate the orbital crossing time in other cases than that of Chambers et al. (1996). We have already started a systematic study on the evolution of protoplanets. The study starts with a reconfirmation of Chambers relation. The final goal is to obtain a theoretical model, as mentioned above. This paper is the first report based on this study.

The aim of this paper is twofold: (1) reconfirmation of the Chambers relation, (2) determination of distribution function of orbital crossing times, not only the average, which is our main purpose. Figure 1 in Chambers et al. (1996) suggests that orbital crossing times for identical initial orbital distance possibly scatter in one order of magnitude range owing to the different initial azimuthal positions of protoplanets. If so, it is very important to confirm the distribution of orbital crossing times in one ensemble for which an initial orbital distance is assigned in order to carry out discussions on the possible accumulation time among protoplanets. In addition, our study on the distribution may provide information on the elemental processes to produce the interesting Chambers relation on the average value among the ensembles with different orbital distance $\Delta$.

We therefore start with a reconfirmation of Chambers law. We explain our numerical simulation in brief in Section 2. This is almost same as that in Chambers et al. (1996). One different point is the number of trial runs executed for one parameter $\Delta$. We carried out 500 runs for each parameter $\Delta$. The reason why we choose this number of samples for one ensemble is explained in Appendix with a short explanation of a method in statistics called "Kolmogorov Smilnov test". In Section 3 we present our numerical results. A comparison between our results and those in Chambers et al. are presented in detail. We can see that both sets of results fit well to each other. Our main result is that the distribution of orbital crossing time is deduced from a number of numerical results. In Section 4 we summarize our results and give the conclusions. Discussions are presented in Section 5.

\section{Basic Equations and Initial Conditions}

The basic equations in this study are the same to those in Chambers et al. (1996). We consider five protoplanets revolving around the Sun. We will put them in sequential number, beginning with the one nearest to the Sun.

Let us suppose the first planet, namely the planet with its orbit nearest to the Sun, has a semi-major axis $a_{0}$, initially. Hereafter we choose $a_{0}$ as a unit of length. For other units, we choose mass of the Sun, $M_{\odot}$, as the unit of mass, and we set the unit of time to make Kepler frequency $\Omega_{0}$ defined as

$$
\Omega_{0}=\sqrt{\frac{\mathrm{G} M_{\odot}}{a_{0}^{3}}}
$$

to be equal to unity.

Using the units mentioned above, the basic equations for the five planets are written as

$$
\frac{d^{2} \mathbf{r}_{\mathrm{i}}}{d t^{2}}=-\frac{\mathbf{r}_{\mathrm{i}}}{r_{\mathrm{i}}^{3}}+\sum_{\mathrm{j} \neq \mathrm{i}}^{5} m \frac{\mathbf{r}_{\mathrm{j}}-\mathbf{r}_{\mathrm{i}}}{\left|\mathbf{r}_{\mathrm{j}}-\mathbf{r}_{\mathrm{i}}\right|^{3}}
$$

where $\mathbf{r}_{\mathrm{i}}$ is a vector from the center of the Sun to the position of the i-th planet and $m$ is mass of one planet. In this equation we neglect the recoil term to the Sun from five planets because their mass is much smaller than unity. In this study the mass of each planet is equal to each other and we choose them to be equal to that in Chambers et al. (1996), which is about equal to the mass of Mercury. Because we choose the mass of the Sun as the unit of mass, we set $m=1 \times 10^{-7}$.

As same as the mass of protoplanets, we choose the initial conditions among them to be the same manner as in Chambers et al. (1996). The semi-major axis of each planet is initially given in the following manner:

$$
a_{\mathrm{i}+1}=a_{\mathrm{i}}+\Delta\left(\frac{2 m}{3}\right)^{1 / 3} \frac{a_{\mathrm{i}+1}+a_{\mathrm{i}}}{2}
$$

In this equation $a_{\mathrm{i}}$ represents the semi-major axis of the i-th planet. As already mentioned, the first planet is the innermost one, and its semi-major axis is chosen as a unit in this study. So, $a_{1}=1$. From this condition and Eq. (3) we can obtain the semi-major axis from second to fifth planets. In Eq. (3), $\Delta$ is an important parameter in this study. We will examine the behavior of planets, placing different initial conditions on $\Delta$. We set it in the range from 4.0 to 8.0 .

All planets initially placed in a co-planer and circular Kepler orbit. So, the initial eccentricities and inclinations are given as

$$
e_{\mathrm{i}}=i_{\mathrm{i}}=0 \text {. }
$$

Because all planets are in co-planer orbits, the inclinations of planets are always equal to zero in this work. We therefore consider 2-D problem only.

As we consider the 2-D problem, we need to specify four orbital elements to determine the initial conditions on each planet. Semi-major axis and eccentricity are given in Eqs. (3) and (4). The argument of perihelion and time of perihelion passage is well known from other orbital elements, though initial argument of perihelion can be set at an arbitrary value, because all planets are in circular orbits. So we put them as

$$
\varpi_{\mathrm{i}}=0 \text {. }
$$

It is more convenient to give the last initial condition as the true anomaly of each planet than as the time of perihelion passage. Here we introduce cylindrical coordinates centered at the position of the Sun, and we represent radial 
and azimuthal components of the i-th planet as $r_{\mathrm{i}}$ and $\theta_{\mathrm{i}}$, respectively. As already mentioned, all planets are in circular orbits initially. Therefore, the radial coordinate of i-th planet is equal to it's semi-major axis. The azimuthal component of the i-th planet is chosen randomly between zero and $2 \pi$.

The parameter $\Delta$ in Eq. (3) is important in this study. We change it from 4.0 to 8.0 at 0.2 intervals. For each case of $\Delta$ we calculate 500 trial runs of the orbital evolution of the five planets and make one ensemble. In each run, different sets of five random numbers are generated to assign the azimuthal components of five planets.

We are now ready for numerical integration. We use the 8-th order Runge-Kutta method with an algorithm for integration of particles' motion in strong external Solar gravity field (Emori et al., 1994).

We pursue the orbital motion of five planets until a pair among them approaches each other. When the distance between two planets becomes shorter than the two body Hill radius, $h$, defined as

$$
h=\left(\frac{2 m}{3}\right)^{1 / 3}
$$

(Nakazawa and Ida, 1988), we stop the calculation and record the time as the crossing time with the value of the parameter $\Delta$. For one trial run, one orbital crossing time is recorded. Because we execute 500 trial runs for one value of $\Delta, 500$ crossing times is obtained for one case of $\Delta$. Using these 500 data points, the average crossing time for $\Delta$ will be presented as in Chambers et al. (1996). In addition, we evaluate the distribution of crossing time around the average value.

\section{Numerical Results}

We first present the time evolution of the orbits for five protoplanets in a case of $\Delta$ equal to 6.0. In Fig. 1 we present the semi-major axis $a_{\mathrm{i}}$ and eccentricity $e_{\mathrm{i}}$ for each planet. Semi-major axes are expressed in Fig. 1(a), the eccentricities are expressed as the distance of the perihelion and aphelion in Fig. 1(b).

We plot $\left(a_{\mathrm{i}}-1\right) / h$ in Fig. 1(a) for five planets given their initial semi-major axis separated every $\Delta(=6.0)$ as functions of time. They revolved around the Sun for about 130,000 times. It takes $2 \pi$ to revolve around the Sun at position of $r=1$ in our units of time and length. After such a long period, a pair of planets, actually the second and third planet in this case, approached each other and the distance between them became shorter than their mutual hill radius defined in Eq. (6). We then recorded the orbital crossing time equal to $1.276 \times 10^{6}$.

Figure 1(a) reveals that the semi major axis of each planet keeps its initial value just before the crossing time. Although semi-major axes of all planets show fluctuations in the last $5 \%$ of total crossing time, the deviations are smaller than the initial orbital separation $\Delta$, which is equal to 6 in the present case. Therefore, we conclude that the orbital crossing between the second and third planets was not brought about by fluctuations in their semi-major axes.

The reason of orbital crossing is well represented in Fig. 1(b). In this figure we plot two values namely $\left(a_{\mathrm{i}}(1+\right.$
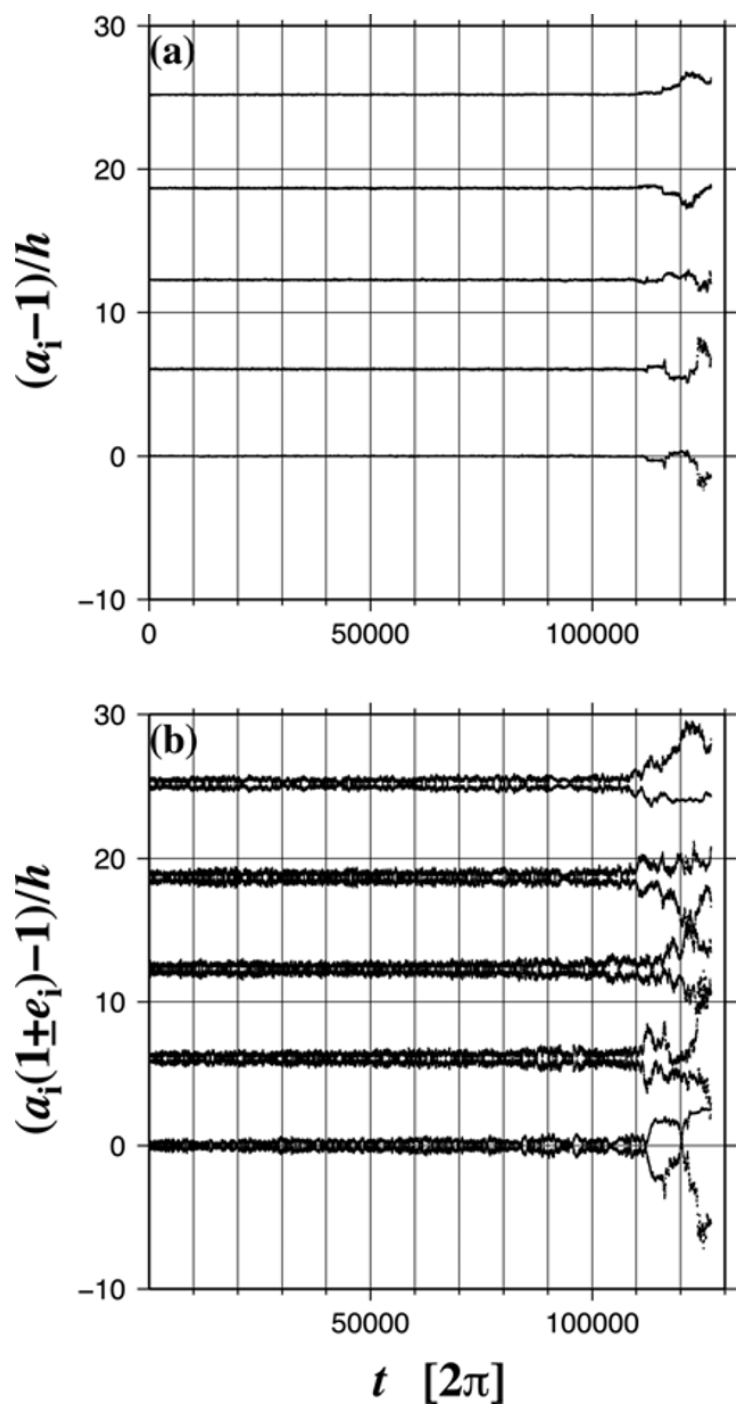

Fig. 1. Orbital evolution of five protoplanets. Mass of a planet and initial distance of semi-major axis between each planet are $1.0 \mathrm{e}-7$ and 6.0 , respectively. In panel (a) semi-major axis of each planet is drawn. In panel (b) radial distance of the perihelion and the aphelion of each planet are presented. Horizontal axis is time in both panels. In horizontal axis time is presented in the unit of $2 \pi$, which is equal to 1 year if the reference orbit is chosen as $a_{0}=1$ [AU].

$\left.\left.e_{\mathrm{i}}\right)-1\right) / h$ and $\left.\left(a_{\mathrm{i}}\left(1-e_{\mathrm{i}}\right)-1\right) / h\right)$, where $h$ is the mutual Hill radius defined in Eq. (6), for each planet as functions of time. The former is the radial distance between the origin of coordinates and the perihelion of $\mathrm{i}$-th planet, and the latter is that of aphelion. So the half of the width between two lines is equal to the eccentricity of each planet. Contrary to the evolution of semi-major axes, the eccentricities of planets increased from an initial value zero continuously with time, though the rates of increase enlarge abruptly at $95 \%$ of the total crossing time. In the last $5 \%$ of crossing time, the eccentricities of planets grow steeply. As a result, a perihelion of a planet moves inward and steps over the aphelion of its inner neighboring planet. In the case of this figure, the two orbits of the second and third planet crosses each other owing to the fact that their eccentricities grow large enough to compensate their initial radial distance $\Delta$.

Iwasaki et al. (2001) have already reported a gradual increase in eccentricities and a sudden jump in a short period 
(a)

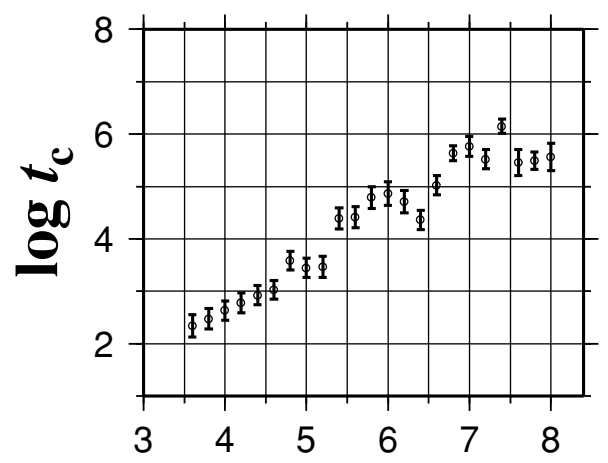

(b)

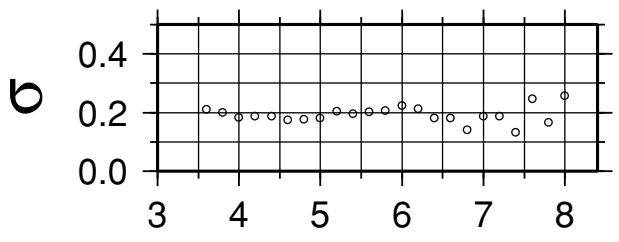

(c)

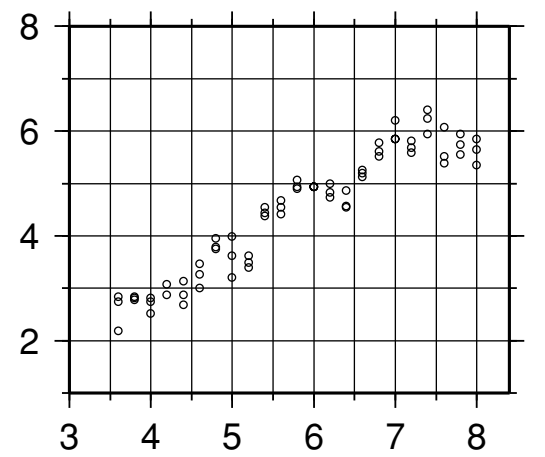

$\Delta$

Fig. 2. In panel (a), averaged orbital crossing time obtained from 500 ensembles for each $\Delta$ is presented with dispersion. Average value and dispersion are defined in Eqs. (7) and (8), respectively. Average value is presented with a circle, and dispersion corresponds to half the length of the vertical bar drawn on the circle. In panel (b), the dispersion is presented as a function of $\Delta$. For comparison, in panel (c) we redraw results presented in Chambers et al. (1996) for the case that five protoplanets each of them has $1 \times 10^{-7} M_{\odot}$ as same as this work.

just before the orbital crossing time. Our results are on common ground about this point.

For one parameter $\Delta$ we carried out 500 trial runs with changing initial azimuthal angle for each planet. In each run we obtain the orbital crossing time in a same manner explained for Fig. 1. Therefore, we obtain 500 different orbital crossing times for one parameter $\Delta$.

In Fig. 2(a) we represent average and dispersion calculated from 500 orbital crossing times for each value of $\Delta$ from 4.0 to 8.0 every 0.2 interval. An average of orbital crossing time is defined as

$$
\left\langle\log \left(t_{\mathrm{c}}\right)\right\rangle_{\Delta}=\frac{1}{500} \sum_{\mathrm{k}=1}^{500} \log \left(t_{\mathrm{c}}(\Delta ; \mathrm{k})\right)
$$

where $t_{\mathrm{c}}(\Delta ; \mathrm{k})$ is an orbital crossing time obtained in the $\mathrm{k}$-th trial run in the ensemble for the case of orbital distance parameter $\Delta$. After the average was obtained, we obtain the dispersion for the case of $\Delta$ as

$$
\sigma(\Delta)=\sqrt{\frac{1}{500} \sum_{\mathrm{k}=1}^{500}\left(\log \left(t_{\mathrm{c}}(\Delta ; \mathrm{k})\right)-\left\langle\log \left(t_{\mathrm{c}}\right)\right\rangle_{\Delta}\right)^{2}} .
$$

In Fig. 2, mean values of orbital crossing time $\left\langle\log \left(t_{\mathrm{c}}\right)\right\rangle_{\Delta}$ is plotted (open circles) and $\sigma(\Delta)$ are represented by half length of each short line stretching upward and downward from each average value point.

We re-confirm the results shown in figure 2 of Chambers et al. (1997) from our Fig. 2(a) and (c). As they pointed out, the average values are well approximated by a linear line on this plane. Therefore, the average of orbital crossing time depends roughly on the parameter $\Delta$ exponentially. Not only this characteristic feature of average orbital crossing time, but also other details shown on the figure, for example positions and magnitudes of many bumps show good agreement to the result in Chambers et al. Iwasaki et al. (2001) studied the orbital crossing time in the case that protoplanets revolve in the gaseous nebula, however, they present the orbital crossing time in the gas-free case for discussion. Comparing their figure (figure 2 in Iwasaki et al. (2001)) provides re-confirmation of the results in Chambers et al.

The number of the trial runs for the initial azimuthal positions of five protoplanets in both Chambers et al. and Iwasaki et al. is equal to five. So, we found that five runs for each case of $\Delta$ is enough to obtain the average value of orbital crossing time. This fact suggests that the distribution functions of orbital crossing time in every case of parameter $\Delta$ may have one sharp peak around each average.

As seen in Fig. 2(a), $\sigma(\Delta)$ are almost equal to each other. We draw $\sigma(\Delta)$ as a function of $\Delta$ in Fig. 2(b). Dispersions of orbital crossing times are equal to 0.2 as long as $\Delta$ is in the range of 4.0 to 5.0. It begins fluctuating as $\Delta$ becomes lower than 5.0. At last $\sigma(\Delta)$ distributes between 0.15 and 0.25 in the range of $\Delta$ between 6.0 and 8.0. Looking at the average orbital crossing times in Fig. 2(a), we recognize that $\left\langle\log \left(t_{\mathrm{c}}\right)\right\rangle_{\Delta}$ can be approximated very well by a line in the range of $\Delta$ smaller than 5.0. Though, as with the fluctuation in the case of $\sigma(\Delta)$, in the range of $\Delta$ greater than $5.0,\left\langle\log \left(t_{\mathrm{c}}\right)\right\rangle_{\Delta}$ begins to deviate from the fitting line. Furthermore, it becomes difficult to approximate the averages of orbital crossing time by one line in the range of $\Delta$ larger than 6.0. To elusidate these behaviors of $\left\langle\log \left(t_{\mathrm{c}}\right)\right\rangle_{\Delta}$ 

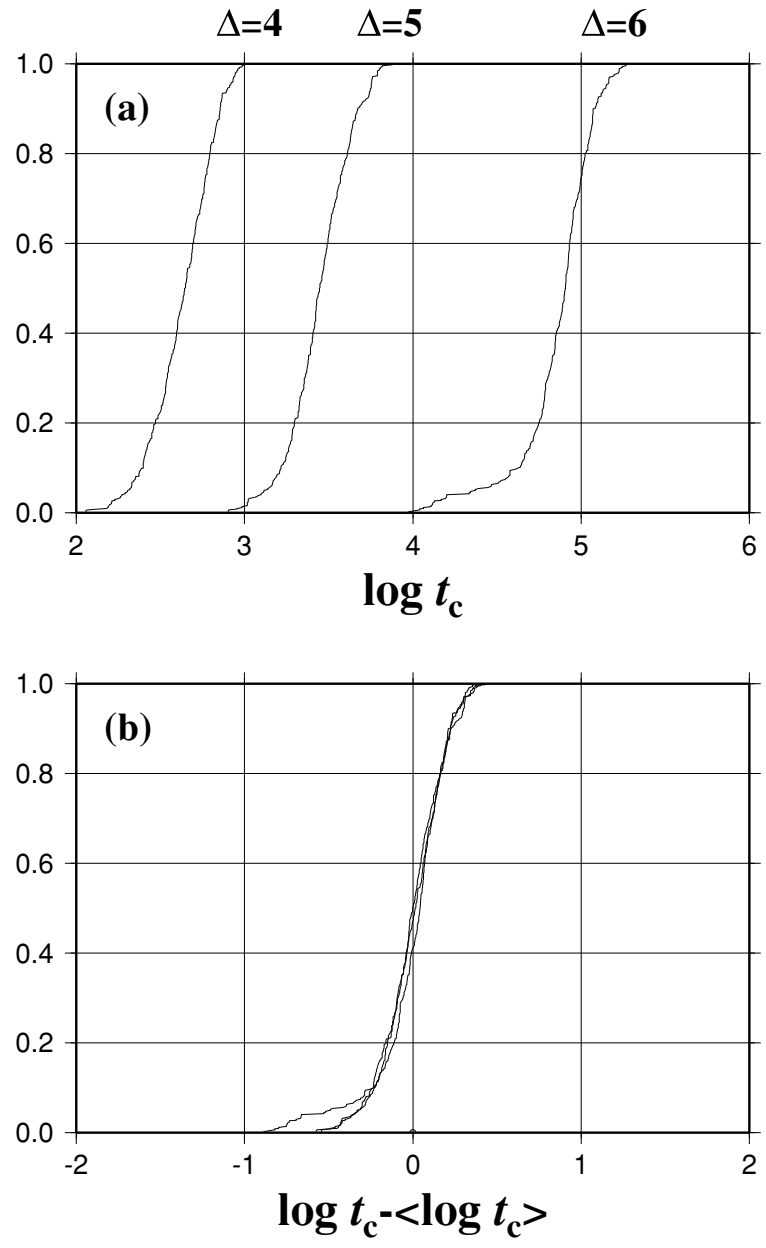

Fig. 3. Comparisons between three distribution functions of orbital crossing time. (a) The distribution for the case of $\Delta$ equal to 4,5 , and 6 are presented in a style of cumulative distribution function. (b) The three distributions are presented too. In this panel, the distribution around mean value of each case is presented. To do so, each distribution is presented for arguments based on the average orbital crossing time.

and $\sigma(\Delta)$, we have to pay attention to the distribution of the orbital crossing time around $\left\langle\log \left(t_{\mathrm{c}}\right)\right\rangle_{\Delta}$ for each value of $\Delta$.

As already shown, the dispersion of the orbital crossing time $\sigma(\Delta)$ is almost equal to 0.2 . If we assume that the distribution of orbital crossing times can be approximated well by the Gaussian distribution function-the validity of this assumption will be discussed later-we can roughly estimate the degree of uncertainties of the orbital crossing time. Namely, the dispersion being equal to 0.2 means that about half of the orbital crossing times distribute in the range of factor 2.25 , namely between $0.63\left(10^{-0.2}\right)$ and $1.6\left(10^{0.2}\right)$ times the average value. More than over about $95 \%$ of orbital crossing times distribute in the range of 6.3 , namely between $0.40\left(10^{-0.4}\right)$ and $2.5\left(10^{0.4}\right)$ times the average value. We had an impression that the dispersion of orbital crossing times could be equal to about one order of magnitude from results in Chambers et al. (1997). However, from this result, we consider that the orbital crossing times may distribute in more narrow width around the average value. To confirm this distribution of the orbital crossing times, let us examine our results more precisely.

Before we present the distribution of orbital crossing times let us explain how we display the distribution. We introduce the probability distribution function $f$ to express the distribution of the orbital crossing time. The probability that we find orbit crossing time between $\log \left(t_{\mathrm{c}}\right)$ and $\log \left(t_{\mathrm{c}}\right)+\mathrm{d} \log \left(t_{\mathrm{c}}\right)$ is expressed as $f\left(\log \left(t_{\mathrm{c}}\right) ; \Delta\right) \mathrm{d} \log \left(t_{\mathrm{c}}\right)$. The distribution function $f$ is normalized as

$$
\int_{-\infty}^{\infty} f(x ; \Delta) \mathrm{d} x=1
$$

where we use $x$ instead of $\log \left(t_{\mathrm{c}}\right)$ as a variable for integration. The parameter $\Delta$ as a argument of $f$ represents that this distribution function is given for the case of orbital separation equal to $\Delta$ times the mutual Hill radius.

We will use another expression of the distribution in many cases. It is called the cumulative distribution function and is defined as

$$
F\left(\log \left(t_{\mathrm{c}}\right) ; \Delta\right)=\int_{-\infty}^{\log \left(t_{\mathrm{c}}\right)} f(x ; \Delta) \mathrm{d} x .
$$

The cumulative fraction tends to zero when $\log \left(t_{\mathrm{c}}\right)$ becomes smaller and smaller, and unity when $\log \left(t_{\mathrm{c}}\right)$ becomes infinite.

In Fig. 3(a) we show the distribution of 500 orbital crossing times for each case of $\Delta$ equal to $4.0,5.0$, or 6.0 in the form of cumulative distribution functions $F$. The horizontal axis is orbital crossing time, namely $\log \left(t_{\mathrm{c}}\right)$, and the vertical axis is $F\left(\log \left(t_{\mathrm{c}}\right) ; \Delta\right)$. We realize that the cumulative distribution changes from zero to unity gradually. The width in which it changes is almost equal to or a little greater than unity in the unit of the horizontal axis. This means that orbital crossing times for a value of $\Delta$ distribute with one order of magnitude width around the average value, although the gradient of cumulative distribution function, which is equal to $f\left(\log \left(t_{\mathrm{c}}\right) ; \Delta\right)$, is not constant. Obviously, for all cases of $\Delta$, the gradient is steepest at the center of the distribution. This means that the distribution of the orbital crossing time $f\left(\log \left(t_{\mathrm{c}}\right) ; \Delta\right)$ has a sharp peak around an average value $\left\langle\log \left(t_{\mathrm{c}}\right)\right\rangle_{\Delta}$. As already mentioned, the dispersion of the distribution is almost equal to 0.2. Therefore, the half width of the distribution is smaller than unity.

Looking at Fig. 3(a) we realize that each distribution has nearly same shape, excepting the difference between average values of crossing times $\left\langle\log \left(t_{\mathrm{c}}\right)\right\rangle_{\Delta}$. To compare distributions around the average value $\left\langle\log \left(t_{\mathrm{c}}\right)\right\rangle_{\Delta}$ we redraw the cumulative distribution with offset $\operatorname{argument} \log \left(t_{\mathrm{c}}\right)-$ $\left\langle\log \left(t_{\mathrm{c}}\right)\right\rangle_{\Delta}$ in Fig. 3(b). This confirms that the distribution functions resemble to each other.

A quantitative comparison between two ensembles on the degree of similarity are possible by the usage of Kolmogorov Smirnov test. The test determines how two ensembles are similar by presenting a numerical value of probabilities. We already have a quantitative comparison using this test on two ensembles, each of which is composed from 500 samples. Details on this test are presented in Appendix. Based these comparisons we draw the following conclusions:

1) The method may give probabilities with one order of magnitude uncertainties. 
Table 1. Probabilities obtained by Kolmogorov Smirnov test comparing ensembles for $\Delta=4.0,5.0$, and 6.0.

\begin{tabular}{ccc}
\hline$\Delta$ & 5.0 & 6.0 \\
\hline 4.0 & $4.60 \mathrm{e}-1$ & $4.16 \mathrm{e}-2$ \\
\hline 5.0 & - & $4.76 \mathrm{e}-3$ \\
\hline
\end{tabular}

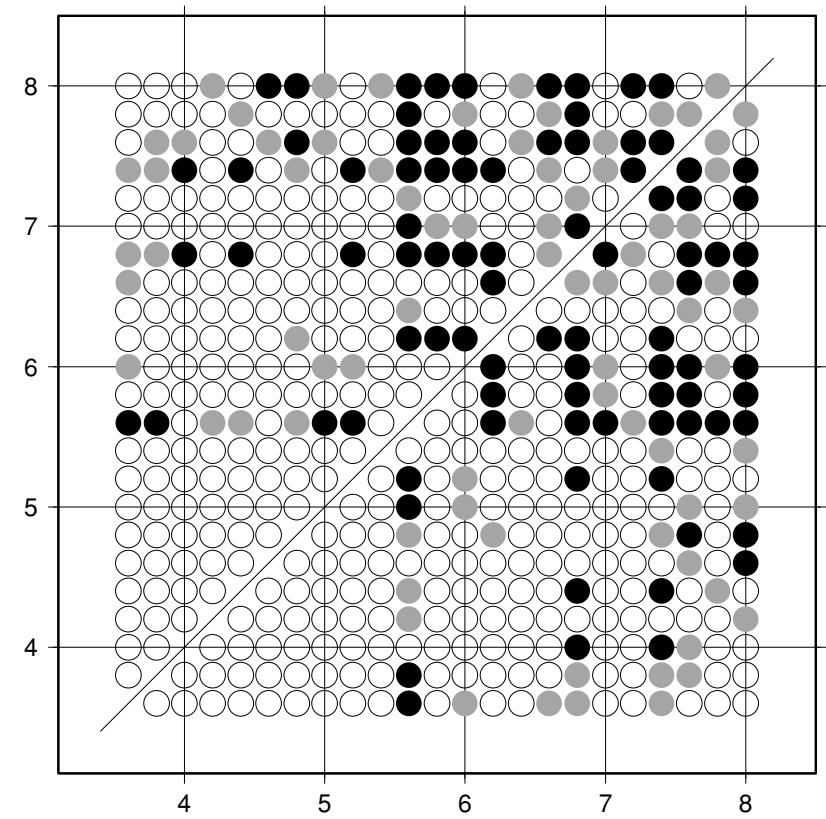

Fig. 4. Comparisons between two distribution functions of orbital crossing time around mean value of each. Result of each pair is presented by open or closed circle plotted on corresponding point on $\Delta-\Delta$ plane.

2) In the case where the number of samples in one ensemble is equal to 500, we can not detect a small difference in two ensembles. If the probability is greater than $1 / 100$, the two distributions are considered to be the same with a precision that the difference is smaller than $30 \%$ of the difference in sigma for two Gaussian distribution functions.

3) When the Kolmogorov Smirnov test gives the probability smaller than $1 / 100$ and larger than $1 / 1000$, two distributions may be different. Though, taking into account the uncertainties of given probability we can not determine completely that they are different distribution.

4) When the Kolmogorov Smirnov test gives the probability smaller than $1 / 1000$, we can determine that the two distributions are different.

We calculated probabilities using the Kolmogorov Smirnov test on the three cumulative distributions in Fig. 3(b). The probabilities are shown in Table 1.

From the results in Table 1 and conclusions in Appendix we evaluate the degree of similarity between the distribution functions of the orbital crossing times around their average value, namely distribution of $\log \left(t_{\mathrm{c}}\right)-\left\langle\log \left(t_{\mathrm{c}}\right)\right\rangle_{\Delta}$ for different values of $\Delta$.

The distributions of orbital crossing time for $\Delta$ equal to 4.0 and 5.0 are the same, because the probability is greater than $1 / 100$. The distributions of $\Delta$ equal to 4.0 and 6.0 are also the same.
The distributions of orbital crossing time for $\Delta$ equal to 5.0 and 6.0 may be different, because the probability is smaller than $1 / 100$. However, we can not exclude completely the possibility that they are same.

In the same manner we compare the orbital crossing times for all cases of $\Delta$ we calculated. To show the probabilities given by the Kolmogorov Smirnov test on the ensembles for two parameters, such as $\Delta_{1}$ and $\Delta_{2}$, we adopt the following approach. If the probability is greater than 0.01 we plot an open circle on the position of $\left(\Delta_{1}, \Delta_{2}\right)$. This open circle means that the difference between two ensembles is smaller than that between two Gaussian distribution functions with $30 \%$ different dispersions.

If the probability is smaller than 0.01 and greater than 0.001 we plot a gray circle on the position. This gray circle means the two ensembles may be different. Finally, if the probability is smaller than 0.001 , we plot a black circle on the position. This black circle means that the two ensembles are different. In Fig. 4 we present the results of comparisons between ensembles of $\log \left(t_{\mathrm{c}}\right)-\left\langle\log \left(t_{\mathrm{c}}\right)\right\rangle_{\Delta}$ for each value of $\Delta$. Horizontal and vertical axes represent $\Delta$. Plots represent probabilities in a manner that has already been explained. Because we plot a mark from our comparison of two ensembles for $\Delta_{1}$ and $\Delta_{2}$ at position $\left(\Delta_{1}, \Delta_{2}\right)$, this figure is symmetric to the diagonal line. To facilitate locating a pair of ensembles to be compared, we plot two plots for one pair of $\Delta_{1}$ and $\Delta_{2}$ on both sides of the diagonal line. We omit to plotting the results of our comparison between identical ensembles.

We plotted 506 $(23 \times 23-23)$ results in Fig. 4. Among these plots, the number of black circles is 86 , and that of gray circles is 78 . So, if we do not argue on that the difference between two ensembles is smaller than $30 \%$ the difference in dispersions between two Gaussian type distributions, we consider 171 pairs of ensemble to belong to the same distribution function, at least. In addition to considering the fluctuation of probabilities given by the Kolmogorov Smirnov test, we can say that 210 pairs of ensemble possibly belong to the same distribution function.

On the 43 pairs of ensembles plotted with a black mark in Fig. 4, we summarize two points. The first is that ensembles of the orbital crossing time in cases that $\Delta$ is greater than 6.8 are different to each other in many cases and are also different to the ensembles with $\Delta$ being smaller than 5.4 in some cases. This is because the dispersions of the ensembles with $\Delta$ being larger than 6.8 show a large fluctuation from the common value in the range of $\Delta<6.6$, as shown in Fig. 2.

The second point to be mentioned on Fig. 4 is about the ensemble of $\Delta$ being equal to 5.6. From Fig. 2, the dispersion, $\sigma(\Delta)$, of this ensemble is equal to 0.2 , though the distribution is different from some ensembles in the range between 3.6 and 5.2. The reason for these discrepancies is not the difference in dispersions. The functional form of the distribution function should be studied to understand the discrepancy. To do that we need the ensembles of a normalized variable, such as $\left(\log \left(t_{\mathrm{c}}\right)-\left\langle\log \left(t_{\mathrm{c}}\right)\right\rangle_{\Delta}\right) / \sigma(\Delta)$.

In Fig. 5 we present results of the Kolmogorv Smirnov test put on two ensembles of $\left(\log \left(t_{\mathrm{c}}\right)-\left\langle\log \left(t_{\mathrm{c}}\right)\right\rangle_{\Delta}\right) / \sigma(\Delta)$ for different $\Delta$. We plotted the probabilities given by the 


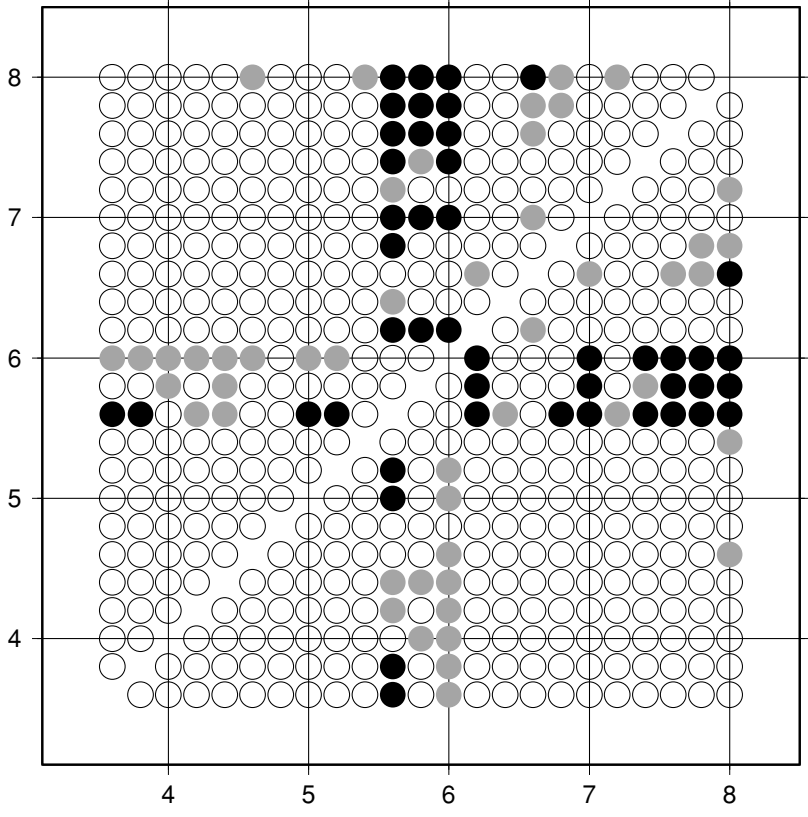

Fig. 5. Comparisons between two distribution functions of normalized orbital crossing time relative to mean value, $\left(\log \left(t_{\mathrm{c}}\right)-\left\langle\log \left(t_{\mathrm{c}}\right)\right\rangle\right) / \sigma(\Delta)$ of each. Result of each pair is presented by open or closed circle plotted on corresponding point on $\Delta-\Delta$ plane.

test in the same manner as that in Fig. 4. As seen from this figure, almost all ensembles belong to the same distribution function. We can find some gray plots, which means that the two ensembles may be different, but we can not determine it completely, even though the number is much smaller than the number of white plots. In addition, it is clear that black plots, which indicate that the two ensembles are different, are plotted only for the three cases of $\Delta$, namely 5.6, 5.8, and 6.0. So we obtain the result that all ensembles possibly belong to the same distribution function, excluding the cases of $\Delta$ equal to $5.6,5.8$, or 6.0 .

We then accumulate 20 ensembles of the normalized value $\left(\log \left(t_{\mathrm{c}}\right)-\left\langle\log \left(t_{\mathrm{c}}\right)\right\rangle_{\Delta}\right) / \sigma(\Delta)$, excluding the cases of $\Delta$ equal to 5.6, 5.8, and 6.0. As a result, an ensemble, including 10000 data was given.

In Fig. 6(a) we present the distribution of orbital crossing time around the average value. The horizontal axis is $\left(\log \left(t_{\mathrm{c}}\right)-\left\langle\log \left(t_{\mathrm{c}}\right)\right\rangle_{\Delta}\right) / \sigma(\Delta)$. The distribution is displayed as a histogram. The distribution function is almost symmetrical to the average value and looks like the Gaussian distribution function, namely

$$
\mathrm{N}(x)=\frac{1}{\sqrt{2 \pi}} \exp \left(-\frac{x^{2}}{2}\right) .
$$

To confirm differences between the Gaussian function defined in Eq. (11) and the distribution displayed in Fig. 6(a), we make a cumulative distribution function from the ensemble of orbital crossing time around the average value, that is

$$
\tilde{F}(x)=\frac{1}{20} \sum_{\Delta} \int_{-\infty}^{\sigma(\Delta) x+\left\langle\log \left(t_{\mathrm{c}}\right)\right\rangle_{\Delta}} f\left(x^{\prime} ; \Delta\right) \mathrm{d} x^{\prime}
$$

where $f(x ; \Delta)$ is the distribution function of orbital crossing time introduced just before Eq. (9). We sum up Eq. (12)
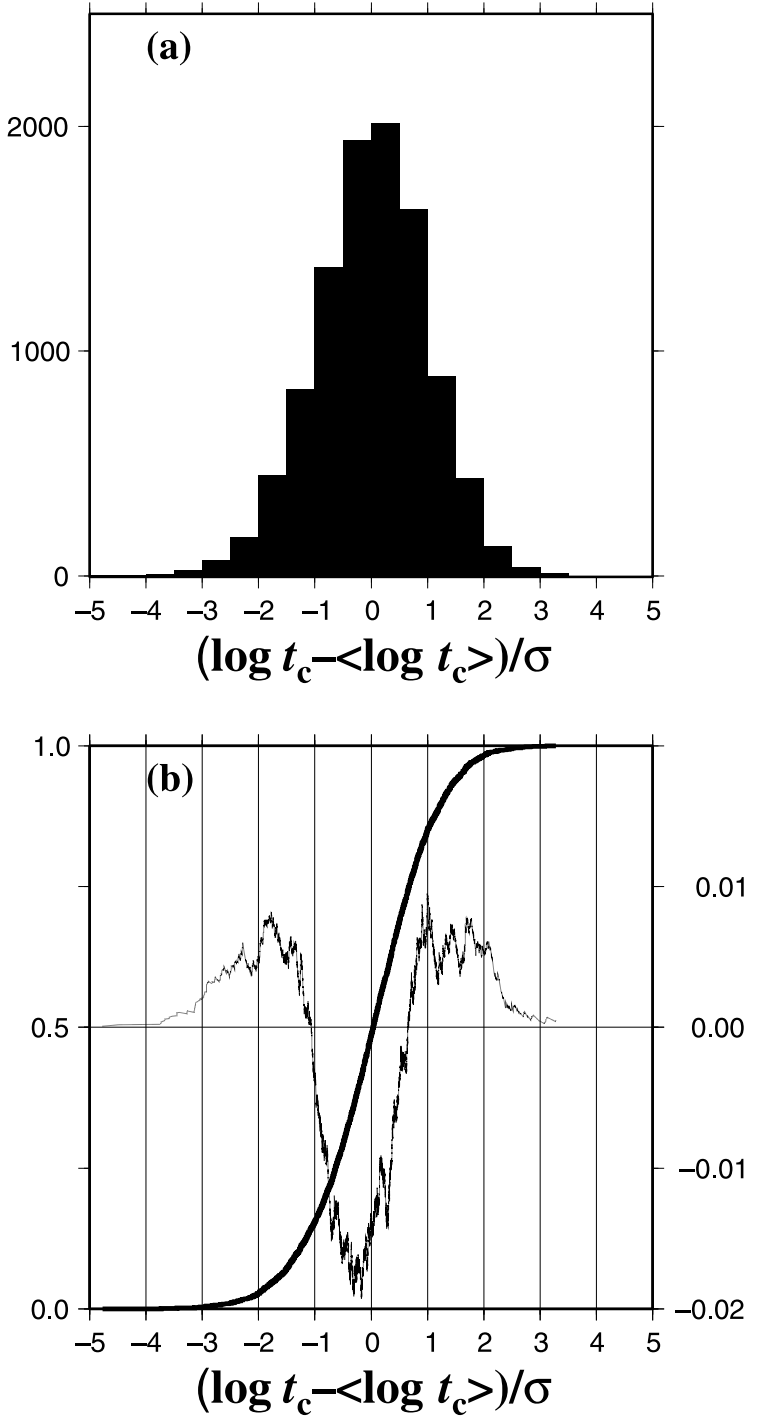

Fig. 6. (a) A Histogram of normalized orbital crossing time relative to mean value, namely $\left(\log \left(t_{\mathrm{c}}\right)-\left\langle\log \left(t_{\mathrm{c}}\right)\right\rangle\right) / \sigma(\Delta)$ composed from 20 ensembles. Each ensemble contains 500 data sets. It looks like a Gaussian distribution. (b) Cumulative distribution functions given from the histogram shown in (a) and difference from cumulative expression of Gaussian distribution function. The thick smooth curve displays the cumulative distribution function. We confirm that it resembles the Gaussian distribution function again from this curve. So, we confirm the difference between them by plotting it with the thin line. The cumulative distribution function should be referred with the vertical axis on the left hand side and the curve of difference from the Gaussian distribution functions should be referred with the vertical axis on the right hand side.

for all $\Delta$ carried out numerical calculations, except for cases of 5.6, 5.8, and 6.0, so that we should obtain a numerical factor equal to $1 / 20$ to normalize this cumulative distribution function. In Fig. 6(b) we present both of $\tilde{F}(x)$ and $\delta \tilde{F}(x)$, which is defined to be the difference between $\tilde{F}(x)$ and the cumulative distribution of Gaussian distribution function, namely

$$
\delta \tilde{F}(x)=\tilde{F}(x)-\operatorname{Erf}(x)
$$

where Erf is the error function, defined as

$$
\operatorname{Erf}(x)=0.5+\int_{0}^{x} \mathrm{~N}\left(x^{\prime}\right) \mathrm{d} x^{\prime}
$$


Horizontal axis $x$ represents a difference in logarithmic orbital crossing time from the average defined in Eq. (7) normalized by the dispersion given in Eq. (8) for each case of $\Delta$. If we need to translate $x$ to orbital crossing time for the case of $\Delta$ we can do it as

$$
\log \left(t_{\mathrm{c}} ; \Delta\right)=\sigma(\Delta) x+\left\langle\log \left(t_{\mathrm{c}}\right)\right\rangle_{\Delta} .
$$

The thick curve smoothly increasing from zero to unity is $\tilde{F}$, and the jagged plot is $\delta \tilde{F}$. The cumulative distribution $\tilde{F}$ should be referred to the left hand side vertical axis; on the contrary, $\delta \tilde{F}$ should be to the right hand one. The curve of $\tilde{F}$ tells us that it is quite similar to the Gaussian distribution. In addition, we can confirm the difference between them with the curve of $\delta \tilde{F}$. The maximum difference is smaller than 0.02 . So we can express the probability of having an orbital crossing event among a system of five equal mass protoplanets in time earlier than time $t$ as follows:

$$
P(t ; \Delta)=\operatorname{Erf}\left(\left(\log (t)-\left\langle\log \left(t_{\mathrm{c}}\right)\right\rangle_{\Delta}\right) / \sigma(\Delta)\right)
$$

where Erf is defined in Eq. (14). So, the probability of having the event at a time between $T$ and $T+\mathrm{d} T$ is expressed as

$$
\left.\frac{\mathrm{d} P(t ; \Delta)}{\mathrm{d} t}\right|_{t=T} \mathrm{~d} T=\mathrm{N}\left(\left(\log (T)-\left\langle\log \left(t_{\mathrm{c}}\right)\right\rangle_{\Delta}\right) / \sigma(\Delta)\right) \mathrm{d} T,
$$

where $\mathrm{N}(x)$ is the Gaussian distribution function defined in Eq. (11).

\section{Conclusion}

Orbital instability time, which is the time needed for the first orbital crossing event between a pair of two protoplanets among five ones, a group of five protoplanets, has been defined as probability distribution functions. In this study, we consider five equal mass protoplanets having co-planar and circular orbits around the Sun. The mass of each planet is equal to $1 \times 10^{-7} M_{\odot}$. These settings of problem is identical to those in Chambers et al. (1996).

We obtain 500 orbital crossing times for one parameter $\Delta$ that is the initial distance between semi-major axes of protoplanets. Randomly chosen initial azimuthal positions were given to each planet in every trial run. From these samples, we calculate average value and dispersion for the distribution of orbital crossing times. In addition, we employ the Kolmogorv Smilnov test to check the distribution of orbital crossing times between each ensembles with different value of $\Delta$. Through this analysis we finally present a model on the probability of the distribution function of orbital crossing times. Our conclusions are as follows:

1) The probability distribution of orbital crossing time is well approximated by the Gaussian distribution function.

2) To evaluate the probability we need both the average $\left\langle\log \left(t_{\mathrm{c}}\right)\right\rangle_{\Delta}$ and the dispersion $\sigma(\Delta)$. The dispersion is almost equal 0.2 in the range that $\Delta<6.6$. This means that about $95 \%$ of orbital crossing times distribute in the range of 0.40 and 2.5 relative to the average orbital crossing time. In the range that $\Delta>6.6$, the dispersion distributes between 1.3 and 2.5. Though the prob- ability distribution function given by Eq. (15) can be used for all cases except for $\Delta=5.6,5.8$, and 6.0.

3) With average $\left\langle\log \left(t_{\mathrm{c}}\right)\right\rangle_{\Delta}$ we once again confirm the conclusion of Chambers et al. (1996). Not only the trend that the logarithmic value of the orbital crossing time is almost proportional to the orbital distance $\Delta$ was reconfirmed, but also detailled features of the averages, such that small but not negligible discrepancies from the fitting line in the region of $\Delta$ greater than 4.8 , were well represented in this work, too.

\section{Discussion}

From the results presented in Chambers et al. (1996) we had the impression that the orbital crossing times could have uncertainties of about one order of magnitude. However, we confirmed that they distribute with a sharper distribution function. As shown in Fig. 6, the distribution function of orbital crossing time, $\log t_{\mathrm{c}}$ is well approximated by the Gaussian distribution function. In addition, the dispersion is almost equal to 0.2 in the range that $\Delta<6.6$. This means that about $95 \%$ of all orbital crossing times distribute in the range of factors associated to the average orbital crossing time between 0.40 and 2.5 . Furthermore, $70 \%$ of these were confined to be in the range between 0.63 and 1.6. So, if we permit $30 \%$ errors, we can say that orbital crossing times among five protoplanets are determined with an uncertainty of factor 2.5 . Moreover, $95 \%$ of the crossing events took place in the range of uncertainties to be about factor 6 .

The average orbital crossing time given for each parameter $\Delta$ was derived from 500 samples. It is very interesting that the averages as a function of orbital distance $\Delta$ fits the results presented by Chambers et al. (1996). This means that it is enough to use only several samples for each $\Delta$ to evaluate an average of them. Needless to say, this rapid convergence is brought by the sharp distribution of orbital crossing times around the average.

From our detailled analysis of average orbital crossing time for each orbital distance $\Delta$, we confirmed that, at some given point of $\Delta$, the average deviates from the fitting line. From Fig. 2, we can say that as the orbital distance $\Delta$ increases, the average orbital crossing time straggles with larger discrepancies from a fitting line, and the dispersion of the distribution $\sigma$ begins to straggle too. As mentioned, our results present a good agreement with the results reported in Chambers et al. (1996) in terms of the behavior of average orbital crossing times. We therefore expect that we should find some physical conditions assignable for the struggling features. The first candidates for the behavior is the resonances between planets.

In this work we examined protoplanets with mass $m=$ $1 \times 10^{-7}$ so mutual hill radius between two protoplanets defined in Eq. (6) is equal to 0.0041. Consequently, the orbital distances between any pair of protoplanets among the group of five ones is very small compared to the reference semi-major axis $a_{1}$, even if the parameter $\Delta$ is equal to 8 . This means that if some kinds of resonance play an important role on the straggling behavior of orbital crossing time, the resonance should be a very high order one. Namely, if a mean motion resonance expressed as $\mathrm{N}: \mathrm{N}+1$ causes the straggling behavior, the number $\mathrm{N}$ must be much larger than 
unity. It is not clear whether such higher order resonances can contribute to the orbital behavior of protoplanets.

Although the distribution of orbital crossing times is presented in this work, there are still some problems in the orbital evolution of protoplanets. The straggling behavior is one of them. Therefore we need more studies on this theme. We have started our studies with one on the protoplanet system with wide range of protoplanet mass parameter. If we consider two systems of protoplanets with three orders of magnitude difference in planets mass, the orbital distance between two protoplanets in each system is different with one order of magnitude, even if the parameter $\Delta$ is equal to each other. The contribution of some kinds of resonance will be examined in this future study as wide mass parameter.

Another future problem is that some kinds of resonance between a protoplanet and other extra perturbing source, namely Jovian planets, are important. In the study of terrestrial planet formation, this effect may be taken as an key process. We have already had a report on the average orbital crossing times that the time is shortened by the extra perturbation (Ito and Tanikawa, 1999). As yet, no studies have been carried out on the distribution of the orbital crossing times under such conditions. It is very difficult to infer the effect of extra perturbations on the distribution function, though we expect that we can do it if we have a theoretical model of orbital evolution of protoplanets, which we will attempt to construct in future studies.

Acknowledgments. The authors have appreciated fruitful discussions with members of the Nakazawa Laboratory, Tokyo Institute of Technology. A part of the numerical simulations carried out in this work were carried out in the GSIC in Tokyo Institute of Technology. This work is supported by a Grant-in-Aid for Scientific Research (C) No. 16540382 brought by Japan Society of the Promotion of Science.

\section{Appendix A. Kolmogorov Smirnov Test}

In this study we use the "Kolmogorov Smirnov test" (Press et al., 1986) to confirm whether two ensembles of orbital crossing times belong to one distribution function or not. Let us explain the procedure in this method briefly. Suppose that there are two distribution functions expressed in a style of the cumulative probability function, each of which is composed from finite number samples. At first we look for the maximum difference between these two distribution functions. Let us express this with $\delta$. Next by the usage of the equation defined as

$$
P=2 \sum_{\mathrm{j}=1}^{\infty}(-1)^{\mathrm{j}} \exp \left(-\left(\frac{\mathrm{N}_{1} \mathrm{~N}_{2}}{\mathrm{~N}_{1}+\mathrm{N}_{2}}\right) \delta^{2} \mathrm{j}^{2}\right)
$$

where $N_{1}$ and $N_{2}$ is number of data in ensemble 1 and 2 , respectively. From this equation we obtain probability $P$.
If $P$ is equal to unity, the two distribution functions are completely same. On the other hand, in the case that $P$ is equal to zero, the two distributions are completely different.

If we compare two ensembles with a finite number of samples, we may never find this Kolmogorov Smirnov test giving the probability being equal to unity. This is because even if two ensembles belong to same distribution function, a finite sample number should bring some differences in two cumulative distribution functions. For example, let us generate two sets of ensembles which are composed from samples brought from a Gaussian distribution function and compare them with the method. The Gaussian distribution function has dispersion $\sigma$ equal to 1.0. The ensembles are as follows;

1) Generate 500 random numbers between 0 and 1 . Let us denote them as $x_{\mathrm{i}}$.

2) For each random number we calculate $v_{\mathrm{i}}$ which satisfies the following condition:

$$
x_{\mathrm{i}}=\left(1+\operatorname{Erf}\left(v_{\mathrm{i}} / 2 \sigma\right)\right) / 2
$$

where Erf is the error function.

Through this procedure with 11 different sets of random numbers, we construct 11 ensembles for the same dispersion $\sigma=1.0$, then we compare the first and each of the other ten ensembles. In Table 2 we present ten probabilities obtained from these comparisons.

As seen from Table 2, the probabilities distribute between 0.67 and 0.029 . From this fact, we must notice that the method gives probabilities with over one order of magnitude uncertainties, even for comparisons between samples with one identical distribution function. So, in a case that we compare two unknown ensembles we can not easily decide the two ensembles belong to different distribution functions even if the probability is smaller than 1.0.

We therefore need some quantitative comparisons between differences in two ensembles and the probabilities given by Kolmogorov Smirnov tests. Let us therefore compare two ensembles selected from two Gaussian distributions with different dispersion $\sigma$. From these data, we present a relation between differences in dispersion and the probability given by Kolmogorov Smirnov test.

Our first step is to construct one ensemble composed from 500 samples randomly selected from a Gaussian distribution function with $\sigma$ equal to 1.0. We call this ensemble our reference ensemble. Next we construct 20 ensembles, each of which is composed of 500 samples randomly selected from a Gaussian distribution function with same $\sigma$ equal to 1.0. We call these ensembles our test ensembles. We compare the reference ensemble and each of the test ensembles, and obtain 20 probabilities. We then obtain the average of these. In the case of a comparison of two ensembles of which the components are sampled from a Gaussian

Table 2. Probabilities obtained by Kolmogorov Smirnov test comparing two ensembles composed from 500 samples randomly sampled from a Gaussian distribution function. First ensemble is compared with from 2 nd to 11 th ensemble.

\begin{tabular}{cccccccccccc}
\hline & 2 & 3 & 4 & 5 & 6 & 7 & 8 & 9 & 10 & 11 \\
\hline 1 & 0.61 & 0.15 & 0.51 & 0.29 & 0.23 & 0.029 & 0.069 & 0.67 & 0.41 & 0.13 \\
\hline
\end{tabular}




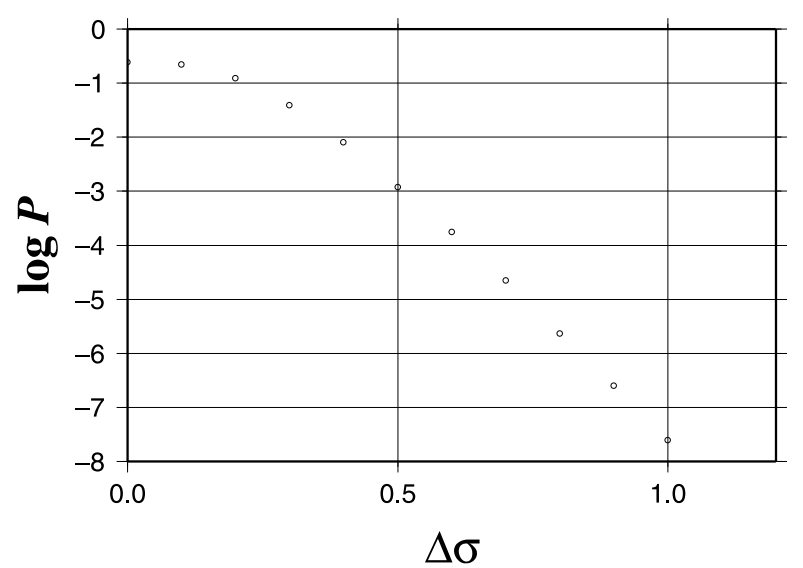

Fig. 7. Quantitative comparison between probabilities given by the Kolmogorov Smirnov test and the difference of distribution functions. Horizontal axis, $\Delta \sigma$ is difference in dispersions of two Gaussian distribution functions. Vertical axis, $\log (P)$ is average probability of two ensembles randomly chosen from the two Gaussian distribution functions. Each ensemble is composed from 500 samples.

distribution function with the same dispersion, the averaged probability is equal to 0.43 . We repeat the generation of test ensembles with changing dispersions from 1.1 to 2.0 for every 0.1 interval and calculate the averaged probabilities by comparing these with reference ensemble. In Fig. 7, we plot averaged probabilities as a function of differences in dispersions between the reference ensemble and test ensembles. Namely, the averaged probability given by the Kolmogorov Smirnov test between the reference ensemble and test ensembles with $\sigma$ equal to 1.1 is plotted as $\Delta \sigma=0.1$. The probabilities are plotted in logarithmic scale.

As shown in Fig. 7 probabilities decreases very steeply as the difference between two dispersion grows. At the same time, it is evident that this test cannot detect small difference in distribution function such that the discrepancies in dispersions of two distribution functions is smaller than $30 \%$, because we have seen from Table 2 that even if two ensembles are sampled from same distribution functions Kolmogorov Smirnov test can give the probability equal to 0.029 , and this probability is equal to average probability on two ensembles sampled from two Gaussian distribution functions which dispersions have $30 \%$ difference.

From these comparison we have following conclusions:

1) The method may give probabilities with one order of magnitude uncertainties.

2) In the case that the number of samples in one ensemble is equal to 500, we can not detect a small difference in two ensembles. If the distribution is Gaussian distribution, the mimimun difference which can be detect by this method is $30 \%$ in sigma. So, if the probability is geater than $1 / 100$, we should the two distribution function may be similar. The word "similar" includes about $30 \%$ error.

3) When Kolmogorov Smirnov test gives the probability smaller than $1 / 100$, the difference beween two distribution function is larger that in two Gaussian distribution functions with difference in dispersions being equal to $30 \%$. Though, taking into account the uncertainties of given probability we can not determine completely that they are different distribution.

4) When Kolmogorov Smirnov test gives the probability smaller than $1 / 1000$, the difference beween two distribution function is larger than that in two Gaussian distribution functions with difference in dispersions being equal to $40 \%$. In this case we can determine that the two distribution is different.

Finaly we must mention that the results presented here is based on comparison two ensembles composed from 500 samples. If in the comparison between ensembles which is composed from more large number of samples, we can not use these results.

\section{References}

Adachi, I., C. Hayashi, and K. Nakazawa, The gas drag effect on the elliptical motion of a solid body in the primordial solar nebula, Prog. Theor. Phys., 56, 1756-1771, 1976.

Chambers, J. E., G. W. Wetherill, and A. P. Boss, The Stability of MultiPlanet Systems, Icarus, 119, 261-268, 1996.

Hasegawa, M. and K. Nakazawa, Distant encounter between Keplerian particles, Astron. Astrophys., 227(2), 619-627, 1990.

Ida, S., Stirring and dynamical friction rates of planetesimals in the solar gravitational field, Icarus, 88, 129-145, 1990.

Ikoma, M., K. Nakazawa, and H. Emori, Formation of giant planets: Dependencies on core accretion rate and grain opacity, Astrophys. J., 537, 1013-1025.

Ito, T. and K. Tanikawa, Stability and Instability of the terrestrial Protoplanet System and Their Possible Roles in the Final Stage of Planet Formation, Icarus, 139, 336-349, 1999.

Iwasaki, K., H. Tanaka, K. Nakazawa, and H. Emori, The Gas-Drag Effect on the Orbital Instability of a Protoplanets System, Publ. Astron. Soc. Jpn., 53, 321-329, 2001.

Iwasaki, K., H. Emori, K. Nakazawa, and H. Tanaka, Orbital Stability of a Protoplanet System under a Drag Force Proportional to the Random Velocity, Publ. Astron. Soc. Jpn., 54(3), 471-479, 2002.

Kokubo, E. and S. Ida, On Runaway Growth of Planetesimals, Icarus, 123(1), 180-191, 1996.

Kokubo, E. and S. Ida, Oligarchic Growth of Protoplanets, Icarus, 131(1), 171-178, 1998.

Mizuno, H., K. Nakazawa, and C. Hayashi, Instability of a gaseous envelope surrounding a planetary core and formation of giant planets, Prog. Theor. Phys., 60, 699-710, 1978.

Nakazawa, K. and S. Ida, Hill's approximation in the three-body problem, Prog. Theor. Phys. Suppl., 96, 167-174, 1988.

Petti, J.-M. and M. Henon, A numerical simulation of planetary rings. IBinary encounters, Astoron. Astrophys., 173(2), 389-404, 1987.

Press, W. H., B. P. Flannery, S. A. Teukolosky, and W. T. Vetterling, Numerical Recipes, Cambridge Univ. Press, London/New York, 1986.

Safronov, V. S., Evolution of the Protoplanetary Cloud and Formation of the Earth and the Planets, Chap. 7, Nauka Press, Moscow, 1969.

Stewart, G. R. and G. W. Wetherill, Evolution of planetesimal velocities, Icarus, 74, 542-553, 1988.

Strom, S. E., S. Edwards, and M. F. Skrutskie, Evolutionary time scales for circumstellar material associated with solar-type pre-main-sequence stars: A possible comstraint on the timescale for planet building, in Protostars and Planets III, edited by E. H. Levy and J. I. Lunine, 837866, University of Arizona Press., Tucson, 1993.

Strom, S. E., Initial frequency, lifetime and evolution of YSO disks, Rev. Mex. Astron. Astrofis, 1, 317-328, 1995.

Yoshinaga, K., E. Kokubo, and J. Makino, The Stability of Protoplanet Systems, Icarus, 139, 328-335, 1999.

Wetherill, G. W. and G. R. Stewart, Accumulation of a swarm of small planetesimals, Icarus, 77, 330-357, 1989.

H. Emori (e-mail: hemori@kuramae.ne.jp), K. Nakazawa, and K. Iwasaki 